\title{
TMEM16A regulates the cell cycle of pulmonary artery smooth muscle cells in high-flow-induced pulmonary arterial hypertension rat model
}

\author{
LIFENG SHANG $^{1}$, KAI WANG ${ }^{2}$, DONGLI LIU ${ }^{1}$, SUYUAN QIN ${ }^{1}$, \\ JINGLIN HUANG ${ }^{1}$, YIJUE ZHAO ${ }^{1}$ and YUSHENG PANG ${ }^{1}$ \\ ${ }^{1}$ Department of Pediatrics, The First Affiliated Hospital of Guangxi Medical University, Nanning, Guanxi 530021; \\ ${ }^{2}$ Department of Pediatrics, The First Affiliated Hospital of Wenzhou Medical University, \\ Wenzhou, Zhejiang 325000, P.R. China
}

Received October 9, 2018; Accepted August 20, 2019

DOI: $10.3892 /$ etm.2020.8589

\begin{abstract}
High-flow-induced pulmonary arterial hypertension (PAH) has attained global notoriety, the mechanism of which remains elusive. The present study investigated the regulation of Anoctamin-1, also known as transmembrane member 16A (TMEM16A), in the cell cycle progression of pulmonary artery smooth muscle cells (PASMCs) from a PAH rat model induced by high pulmonary blood flow. A total of 30 Sprague-Dawley rats were randomly assigned into control, sham and shunt groups. A rat model of high pulmonary blood flow-induced PAH was established by surgery using abdominal aorta-inferior vena cava fistula. Right ventricular pressure, right ventricular hypertrophy index and pulmonary arteriole structural remodeling were assessed 11 weeks following operation. The cell cycle statuses of PASMCs was assessed via flow cytometry, whereas western blot analysis was performed to measure the expression of cyclin D1, CDK2, p27KIP and cyclin E in primary PASMCs isolated from rats. The expression of cyclin E and cyclin D1 was revealed to be increased in the shunt group compared with the control group, which was accompanied with an increased expression of TMEM16A in the shunt group. Changes in the ratio of PASMCs in the $G_{0} / G_{1}, S$ and $G_{2} / M$ phases of cycle induced by PAH were reversed by TMEM16A knockdown. The expression of cyclin $\mathrm{E}$ and cyclin D1 in the shunt group was significantly higher compared with the control group in vitro, which was reversed by TMEM16A-siRNA transfection. In conclusion, TMEM16A may be involved in high pulmonary blood flow-induced PAH by regulating PASMC cell cycle progression.
\end{abstract}

Correspondence to: Professor Yusheng Pang, Department of Pediatrics, The First Affiliated Hospital of Guangxi Medical University, 6 Shuangyong Road, Nanning, Guangxi 530021,P.R. China E-mail:pangyush@163.com

Key words: transmembrane member 16A, pulmonary artery smooth muscle cells, cell cycle, pulmonary arterial hypertension, congenital heart disease

\section{Introduction}

Congenital heart disease (CHD) is one of the most common birth defects in children. The mean prevalence of CHD from 1970 to 2017 stood at 8.224 per 1,000 globally (1). Furthermore, the estimated incidence of sustained $\mathrm{PH}$ in all categories was reported at 5-8 cases per million children per year and 26-33 per million children in the USA (2). In particular, left-to-right shunt CHD is the most common form of CHD. Due to long-term high pulmonary blood flow, endothelial dysfunction and structural remodeling ensues in the pulmonary arterioles from high vascular shearing force, which ultimately leads to elevated arterial pressure and resistance in the pulmonary vasculature (3). Pulmonary artery smooth muscle cells (PASMCs) serve a vital role in vascular homeostasis, the regulation of vascular tone and the regulation of pulmonary arterial pressure. As one of the main transmembrane channels, calcium-activated chloride channels (CaCCs) serve an important function in transmembrane chloride ion homeostasis and vascular tone regulation (4-6). As PAH progresses, increased proliferation of smooth muscle cells, endothelial cells and adventitial fibroblasts, coupled with reduced PASMCs apoptosis contribute to the medial thickening and neomuscularization of distal arterioles, ultimately resulting in right ventricular hypertrophy and heart failure (7).

Transmembrane protein 16A (TMEM16A, also known as ANO1) was first reported to encode CaCCs in 2008 (8-10). TMEM16A is expressed in a variety of cells, including cardiac fibroblasts (11), smooth muscle cells (12) and sympathetic ganglion cells (13). TMEM16A has been revealed to be overexpressed in PASMCs in a number of different pulmonary hypertension models $(14,15)$. A previous study from our laboratory at the Pediatric department, The First Affiliated Hospital Of Guangxi Medical University (Nanning, China) demonstrated that the $\mathrm{CaCC}$ inhibitor, niflumic acid, attenuated pulmonary artery structural remodeling in PAH induced by high pulmonary blood flow in vivo (16). However, the role and mechanism of TMEM16A in PAH induced by high pulmonary blood flow remains unclear. Therefore, the present study investigated the effects of TMEM16A in the regulation of PASMCs in high pulmonary blood flow-induced PAH. 


\section{Materials and methods}

Animals and PAH models. All animal experimental procedures were performed in accordance with the Guide to Care and Use of Experimental Animals issued by the Ministry of Health of the People's Republic of China. All experimental procedures were approved by The Animal and Human Ethics Committee in Guangxi Medical University (Guangxi, China). A total of 30 male Sprague Dawley rats (weight, 180-200 g; age, 6-8 weeks) were provided by the Animal Research Centre of Guangxi Medical University (license no. SCXK 2009-0002). A total of 10 rats were randomly assigned into three groups respectively: Control, sham and shunt groups. Rats were anaesthetized by intraperitoneal injection of sodium pentobarbital $(0.25 \% ; 40 \mathrm{mg} / \mathrm{kg})$. According to methods reported previously $(16,17)$, an abdominal aorta and inferior vena cava arteriovenous fistulization was performed to establish PAH induced by high pulmonary blood flow from the systemic circulation. Laparotomy was performed in the sham and shunt groups, and the abdominal aorta was clamped for $3 \mathrm{~min}$. All rats had were housed in a specific pathogen free room with free access to food and water, maintained at $22-24^{\circ} \mathrm{C}$ with $40 \%$ humidity and $12 \mathrm{~h}$ light/dark cycle.

Right ventricular $(R V)$ pressure $(R V P)$, right ventricular hypertrophy index (RVHI) and pulmonary structural remodeling measurements. RVP, including systolic right ventricular pressure (SRVP), diastolic right ventricular pressure (DRVP) and mean right ventricular pressure (MRVP) were measured 11 weeks after surgery using a cardiac function analyzer (MP160; Bipoac Systems, Inc.) as previously described (16,17). Weights of the RV and left ventricle (LV) with the septum (S) of the hearts were measured after sacrifice. RVHI was calculated using the following formula: $\mathrm{RVHI}=(\mathrm{RV}) /(\mathrm{LV}+\mathrm{S})$. Pulmonary arteriole tissue was isolated and fixed in 10\% paraformaldehyde at room temperature (RT) for $2 \mathrm{~h}$, dehydrated in a graded alcohol series, cleared with xylene and embedded in paraffin. Tissue was then cut into $5 \mu \mathrm{m}$ sections. Hematoxylin and eosin (H\&E) staining was performed to observe pulmonary structural remodeling. All slides were imaged using a video-linked light microscope (magnification x100; Olympus CX31; Olympus Corporation) and analyzed using the ImageJ software (version 1.49p; National Institutes of Health).

PASMC isolation and culture. Primary PASMCs from control, sham and shunt groups were isolated and cultured for in vitro assessment as previously described $(16,17)$. Pulmonary arteriole tissue was removed and immersed in ice-cold HEPES-buffered salt solution. The endothelium was removed by rubbing the luminal surface using a cotton swab. Tissue was then cut into $1 \times 1 \mathrm{~mm}$ pieces and the arterioles were digested at $37^{\circ} \mathrm{C}$ for $20 \mathrm{~min}$ in HBSS containing $20 \mu \mathrm{mol} \mathrm{Ca}{ }^{2+}, 1,750 \mathrm{U} / \mathrm{ml}$ type I collagenase, $9.6 \mathrm{U} / \mathrm{ml}$ papain, $20 \%$ fetal bovine serum (FBS; Thermo Fisher Scientific, Inc.) and $1 \mathrm{mM}$ dithiothreitol. A total of $3-4 \times 10^{4}$ cells were seeded into a $25 \mathrm{~T}$ culture flask and placed in a $5 \% \mathrm{CO}_{2}$ incubator, maintained at $37^{\circ} \mathrm{C}$. The culture medium with DMEM containing $10 \%$ FBS was refreshed 5 days following culture and $\sim 80 \%$ confluence was typically achieved on day 9 , where cell passage was performed until the passage nine.
Immunocytochemical staining. PASMCs were trypsinized and suspended using a pipette onto a circular coverslip in a 6-well plate. After rinsing with PBS three times, PASMCs were fixed with 4\% paraformaldehyde for $15 \mathrm{~min}$ at RT. Following permeabilization in a $0.2 \%$ Triton X-100 solution, $1 \%$ bovine serum albumin (BSA; Sigma-Aldrich; Merck KGaA) was used for blocking for $30 \mathrm{~min}$ at RT. The coverslips were incubated with primary antibodies against $\alpha$-actin (1:100, cat. no. sc-32251; Santa Cruz Biotechnology, Inc.) for $8 \mathrm{~h}$ at RT. Secondary antibodies were subsequently added in a dropwise manner, and the mixture was incubated at room temperature for $30 \mathrm{~min}$. The coverslips were then incubated with diaminobenzidine, counterstained with hematoxylin for $5 \mathrm{sec}$ at RT and differentiated using hydrochloric acid/alcohol before being sealed. A video-linked light microscope (magnification, x100; Olympus CX31; Olympus Corporation) was used for imaging the coverslips.

Lentiviral siRNA infection of PASMCs. Lentiviruses (LV-TMEM16A-siRNA and control siRNA) enconding green fluorescence protein (GFP) were purchased from Shanghai Jikai Gene Chemical Technology Co., Ltd., and PASMCs were transfected as previously described (17). Puromycin was used for cell screening. PASMCs from the shunt group were digested with $0.25 \%$ trypsin and suspended at a density of $2 \times 10^{4} / \mathrm{ml}$ prior to seeding into a 6 -well plate on day 1 after culture. Lentiviral particles prepared in a $2 \mathrm{ml}$ suspension were then added after $24 \mathrm{~h}$ to achieve a multiplicity of infection of 80 with the final concentration of Polybrene maintained at $5 \mu \mathrm{g} / \mathrm{ml}$. The culture solution was changed after $36 \mathrm{~h}$ and cells were cultured in a $37^{\circ} \mathrm{C}, 5 \% \mathrm{CO}_{2}$ incubator for a further 3 days before subsequent experiments. For in vitro studies, PASMCs were divided into control, shunt, shunt + siRNA-TMEM16A and shunt + siRNA-negative control groups.

Flow cytometry. When the cells grew to cover $~ 85 \%$ of the flask, they were harvested, centrifuged ( $5 \mathrm{~min}, 175 \mathrm{x} \mathrm{g}$ ) at RT and washed twice with $3 \mathrm{ml}$ PBS. 70\% ethanol was used for cell fixation for $1 \mathrm{~h}$ at $4^{\circ} \mathrm{C}$. Cells were then centrifuged at $850 \mathrm{xg}$ for $5 \mathrm{~min}$ at RT and washed twice with $3 \mathrm{ml}$ PBS. Propidium iodide (PI; $500 \mu \mathrm{l}$ ) was added for staining according to the manufacturer's protocol (cat. no. 550825; BD Biosciences). The proportions of cells in $G_{0} / G_{1}$ phase, $S$ and $G_{2} / M$ phases were measured using flow cytometry $\left(\right.$ Guava $^{\circledR}$ easyCyte $^{\mathrm{TM}}$ 6-2L; Merck KGaA) and the guavaSoft ${ }^{\mathrm{TM}}$ software (version 3.1.1; Merck KGaA).

Western blotting. Proteins were homogenized or lysed in ice-cold RIPA lysis buffer (Santa Cruz Biotechnology, Inc.). The concentration of the protein was examined using Bradford protein assay kit (Bio-Rad Laboratories, Inc.). In total, $20 \mu \mathrm{g}$ proteins were separated by $10 \%$ sodium dodecyl sulfate polyacrylamide gel electrophoresis (SDS-PAGE) and transferred to nitrocellulose membranes (Bio-Rad Laboratories, Inc.). The membranes were blocked in Tris-buffered saline with $5 \%$ non-fat milk and $0.5 \%$ BSA for $1 \mathrm{~h}$ at RT as previously described (17). Membranes with corresponding proteins were incubated with primary antibodies against TMEM16A (1:1,000; cat. no. ab53213; Abcam), cyclin D1 (1:1,000; cat. no. 2978; Cell Signaling Technology, Inc.), cyclin-dependent 

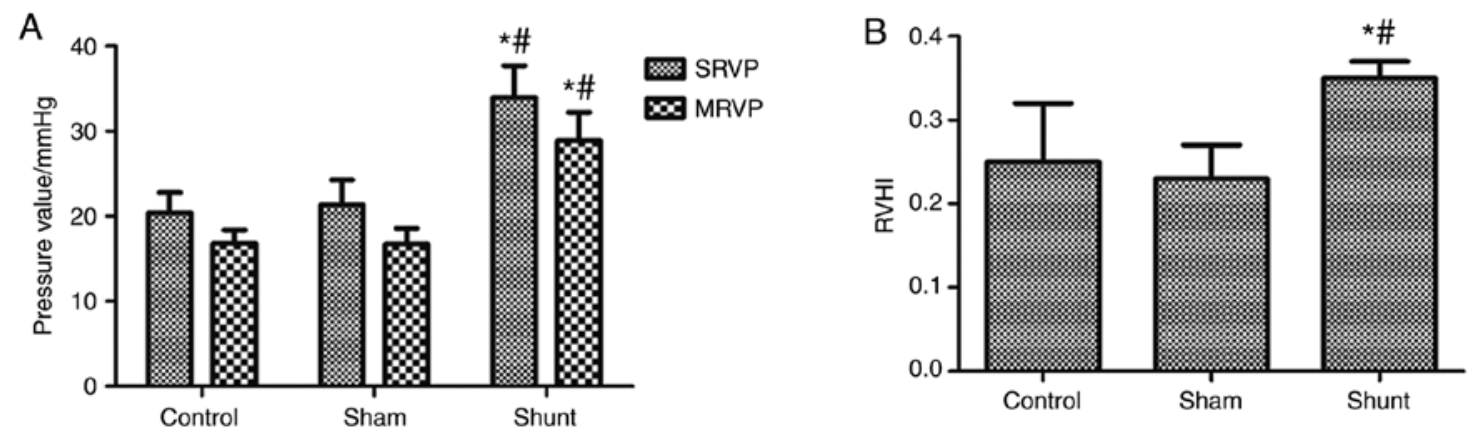

Figure 1. High pulmonary blood flow-induced pulmonary hypertension leads to elevated right ventricular pressure and RVHI. (A) SRVP and MRVP, and (B) RVHI was measured in rats from the control, sham and shunt groups. Data are presented as the mean \pm standard deviation $(\mathrm{n}=10)$. ${ }^{*} \mathrm{P}<0.05$ vs. control and ${ }^{\#} \mathrm{P}<0.05$ vs. sham. RVHI, right ventricular hypertrophy index; SRVP, systolic right ventricular pressure; MRVP, mean right ventricular pressure.
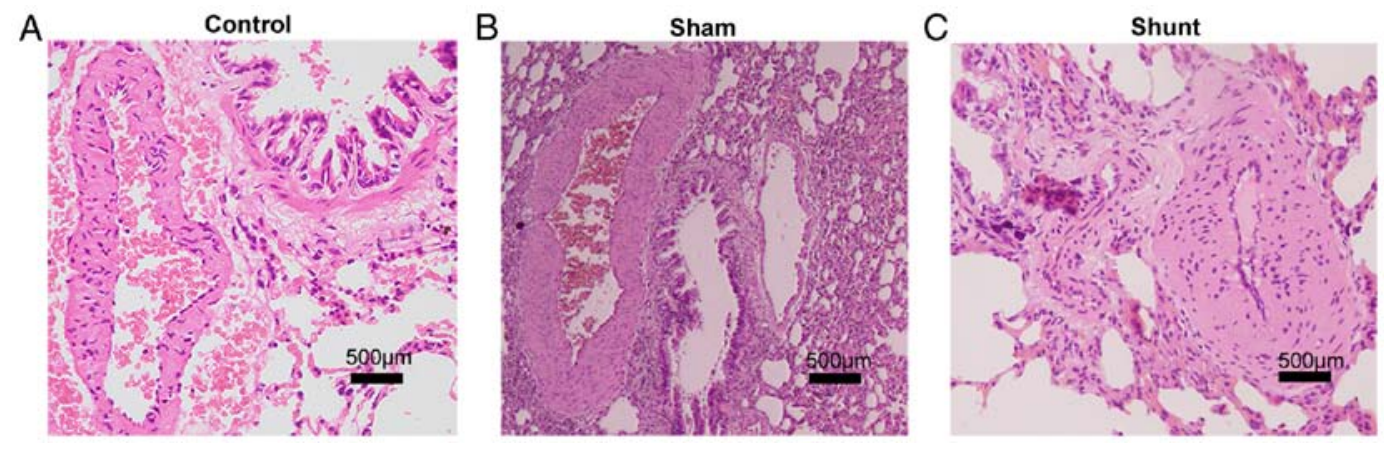

Figure 2. Pathological changes of pulmonary arterioles by H\&E staining. Images of H\&E staining of pulmonary arteriole tissues of rats from (A) control, (B) sham, and (C) shunt groups (magnification, x100).

kinase 2 (CDK2; 1:1,000; cat. no. 2546; Cell Signaling Technology, Inc.), cyclin-dependent kinase inhibitor P27 (p27KIP; 1:1,000; cat. no. 3686; Cell Signaling Technology, Inc.), cyclin E1 (1:1,000; cat. no. 20808; Cell Signaling Technology, Inc.) and $\beta$-actin (1:5,000, cat. no. 21338; SAB Biotherapeutics, Inc.) overnight at $4^{\circ} \mathrm{C}$. The membranes were then incubated with a horseradish peroxidase-conjugated goat anti-rabbit IgG secondary antibody (1:5,000; cat. no. L3012, SAB Biotherapeutics, Inc.) for $1.5 \mathrm{~h}$ at RT, and washed four times using $0.1 \%$ Tween-20 solution. Blots were visualized with SuperSignal ${ }^{\mathrm{TM}}$ West Femto Maximum Sensitivity Substrate (Thermo Fisher Scientific, Inc.) and quantified with Quantity 5.2 software System (Bio-Rad Laboratories, Inc.).

Statistical analysis. SPSS 22.0 software (IBM Corp.) was used to analyze the data. Data are expressed as the mean \pm standard deviation. A two-sample t-test was used for comparisons between two groups. One-way ANOVA was used for comparisons between groups followed by Turkey's multiple comparisons. All hypotheses were tested bilaterally. $\mathrm{P}<0.05$ was considered to indicate a statistically significant difference.

\section{Results}

Establishment of high blood flow-induced PAH and structural remodeling. After 11 weeks of shunting, SRVP and MRVP were significantly higher in the shunt group compared with the sham group, whereas no statistically significant differences were observed between the control and sham groups (Fig. 1A). Similarly, RVHI in the shunt group was significantly higher compared with the control and sham groups, but no significant differences were observed in the RVHI between the control and sham groups (Fig. 1B). Histological staining demonstrated marked thickening of the pulmonary arteriole walls in the shunt group that was accompanied with narrowing of the lumen, which was not observed in the control and sham groups (Fig. 2).

Characteristics and transfection of lentiviral TMEM16A-siRNA transfection in PASMCs. Cultured PASMCs in all three groups (Control, sham and shunt) grew and aggregated at 3-5 days after seeding (Fig. 3A), and colonies formed on $\sim$ day 10 . Typically, spindle-shaped cells start to divide and extend on the culture plate in a dispersed pattern, with some colonies forming clusters (Fig. 3B). Immunocytochemical staining confirmed the expression of $\alpha$-smooth muscle actin based on the specific myofilament structure of PASMCs (Fig. 3C). A lentiviral vector specifically expressing TMEM16A-siRNA or the corresponding scramble control vector was transfected into PASMCs from the shunt group. Cells were screened using $3 \mathrm{mg} / \mathrm{l}$ puromycin and GFP fluorescence was observed using an inverted fluorescence phase contrast microscope (Fig. 3D). Flow cytometry was also used to measure lentiviral transfection efficiency, which was found to be $>80 \%$ (data not shown).

TMEM16A expression is upregulated in PASMCs following $P A H$ induction. The expression of TMEM16A in PASMCs 

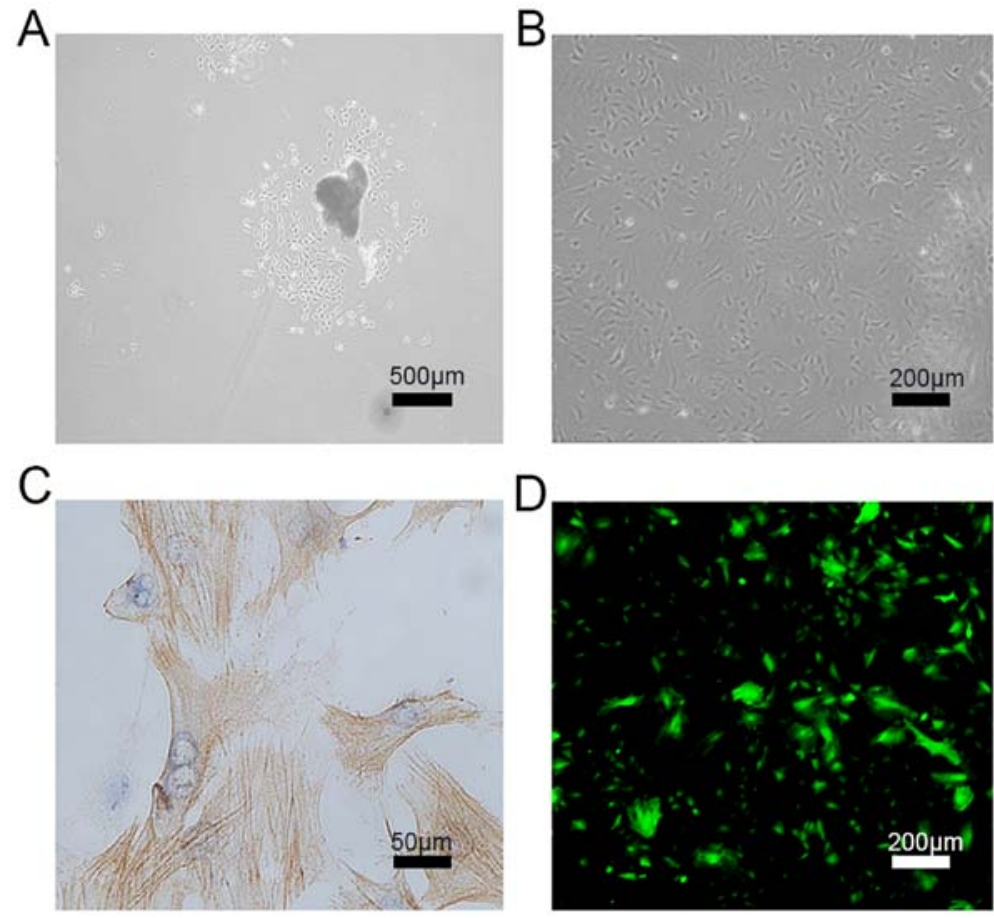

Figure 3. Characterization of primary PASMCs isolated from pulmonary arteries of rats. (A) PASMCs isolated from the pulmonary artery mass and aggregated into clusters ( 5 days after start of culture). (B) PASMCs exhibiting long fusiform shapes and 'peak-valley' appearance (10 days after start of culture). (C) Immunocytochemistry staining confirmed $\alpha$-smooth muscle actin expression based on the specific myofilament structure of PASMCs (15 days after start of culture). (D) Specific TMEM16A-siRNA lentiviral vectors, which also encode GFP, were transfected into PASMCs and observed under a light microscope. PASMCs, pulmonary artery smooth muscle cells; TMEM16A, transmembrane member 16A; siRNA, small interfering RNA.
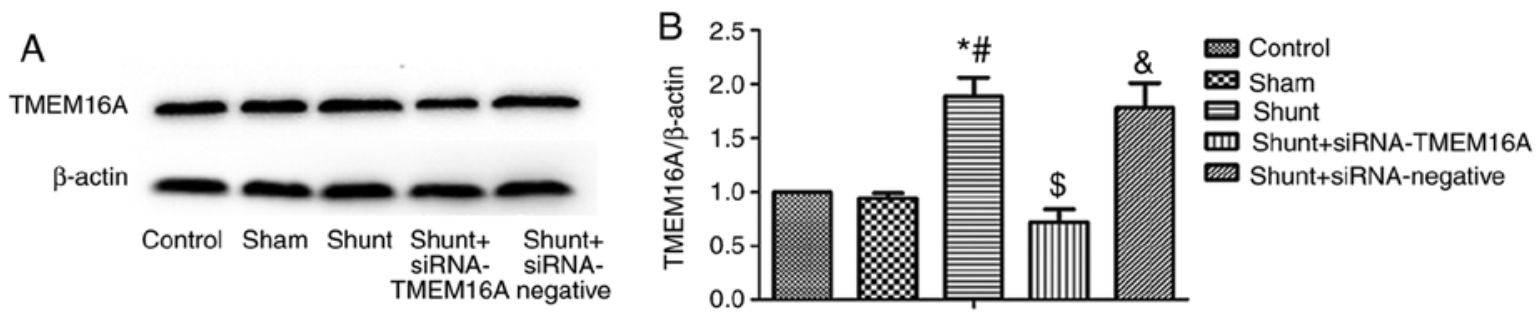

Figure 4. TMEM16A protein expression in primary PASMCs isolated from rats of the control, sham and shunt groups, in addition to PASMCs from the shunt group transfected with TMEM16A-siRNA. (A) Western blotting of TMEM16A and $\beta$-actin in primary PASMC samples from rats of the various experimental groups. (B) Quantified densitometry results of (A). Data are presented as mean \pm standard deviation ( $\mathrm{n}=10)$. " $\mathrm{P}<0.05$ vs. control; $\mathrm{P}<0.05$ vs. sham; ${ }^{\$} \mathrm{P}<0.05$ vs. shunt; and ${ }^{\circledR} \mathrm{P}<0.05$ vs. shunt + siRNA-TMEM16A. TMEM16A, transmembrane member 16A; PASMCs, pulmonary artery smooth muscle cells; siRNA, small interfering RNA.

was significantly higher in the shunt group compared with the control and sham groups, whereas no significant differences were observed between the control and sham groups (Fig. 4A and B). Transfection of PASMCs with lentiviral TMEM16A-siRNA significantly reduced TMEM16A expression in the shunt group compared with non-transfected cells from the same group, but not in cells transfected with the scramble control siRNA (Fig. 4A and B).

TMEM16A may regulate cell cycle progression in PASMC following PAH induction. To examine the TMEM16A-mediated regulation of PASMC cell cycle progression, the ratios of cells in $\mathrm{G}_{0} / \mathrm{G}_{1}, \mathrm{~S}$ and $\mathrm{G}_{2} / \mathrm{M}$ phases were measured via flow cytometry. The ratio of cells in the $\mathrm{G}_{0} / \mathrm{G}_{1}$ phase was significantly decreased in PASMCs in the shunt group compared with the control group, and TMEM16A-siRNA abolished this increase (Fig. 5A and B).
The ratios of $\mathrm{S}$ and $\mathrm{G}_{2} / \mathrm{M}$ in PASMCs from the shunt group was significantly increased compared with the control group, which was also reversed by TMEM16A-siRNA transfection but not by the negative control siRNA (Fig. 5A and B). These data suggest that TMEM16A may be involved in cell cycle progression regulation in PAH induction.

TMEM16A contributes to the regulation of proteins associated with the PASMC cell cycle. To clarify the potential mechanism of TMEM16A in PAH induced by high blood flow, the expression of proteins associated with cell cycle regulation, including cyclin D1, cyclin E, CDK2 and p27KIP, in PASMCs from the different experimental groups was detected. The expression of cyclin D1 and cyclin E in the shunt group was significantly higher compared with the control group, which was reversed by TMEM16A-siRNA transfection. There were no significant differences in CDK2 or p27KIP expression 

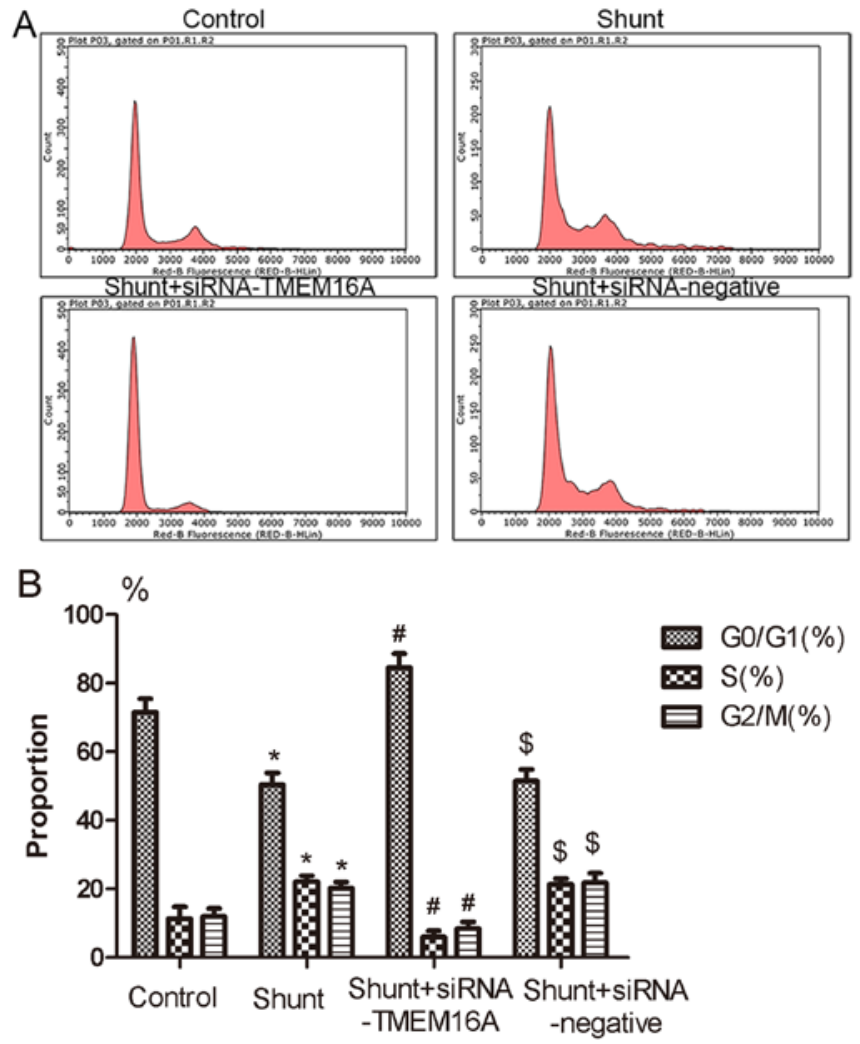

Figure 5. PASMC cell cycle progression as regulated by TMEM16A in high blood flow induced by PAH. (A) Representative FACS dot plots presenting cell cycle distribution of primary rat PASMCs from the four indicated experimental groups. (B) Quantified results of (A) presenting the proportion of primary rat PASMCs in $\mathrm{G}_{0} / \mathrm{G}_{1}, \mathrm{~S}$ and $\mathrm{G}_{2} / \mathrm{M}$ phases from the four indicated experimental groups. Data are expressed as the mean \pm standard deviation $(\mathrm{n}=10)$. ${ }^{*} \mathrm{P}<0.05$ vs. control; ${ }^{\#} \mathrm{P}<0.05$ vs. shunt; ${ }^{\$} \mathrm{P}<0.05$ vs. shunt + siRNA-TMEM16A. PASMCs, pulmonary artery smooth muscle cells; TMEM16A, transmembrane member 16A; siRNA, small interfering RNA.

between the control and shunt groups (Fig. 6). Taken together, the results indicated that TMEM16A might contribute to the regulation of proteins associated with cell cycle progression in PASMCs.

\section{Discussion}

High pulmonary blood flow-induced PAH is one of the most common causes of PAH during childhood, the mechanism of which has not been fully elucidated. Under aberrant levels of mechanical shear stress caused by excessive blood flow, pulmonary arteriole endothelial and smooth muscle cells undergo progressive dysfunction. A previous study $(16,18)$ in our laboratory in the Pediatric department, The First Affiliated Hospital of Guangxi Medical University (Nanning, China) demonstrated that $\mathrm{CaCCs}$ contribute to elevated pulmonary artery pressure and structural remodeling in PAH induced by high pulmonary flow. TMEM16A was reported as a candidate for the molecular basis of CaCCs in 2008 (8-10), and there is accumulating evidence revealing that TMEM16A is involved in a variety of physical and pathophysiological processes $(15,19-22)$. However, the mechanism of TMEM16A regulation of PASMCs in PAH induced by high blood flow remains unclear. The present study established an animal model of high blood flow-induced PAH, which increased TMEM16A expression accompanied with
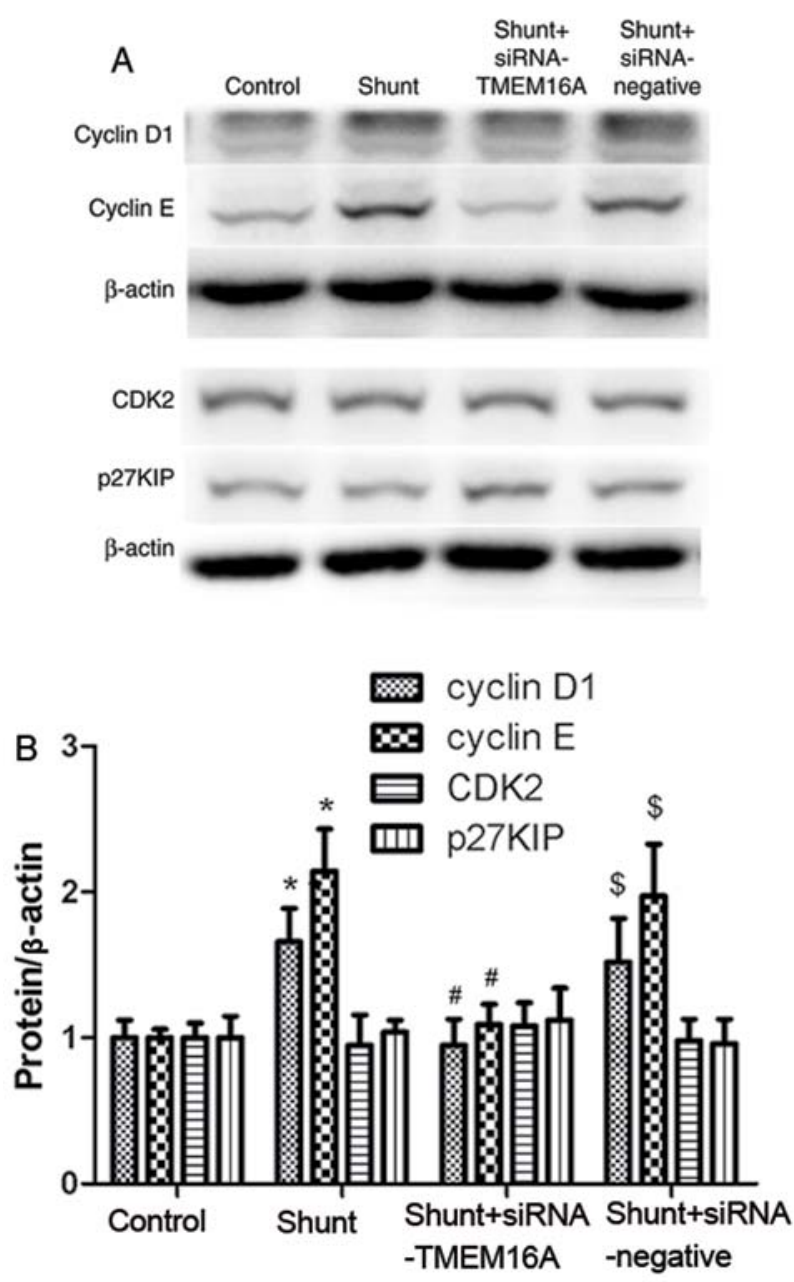

Figure 6. TMEM16A contributed to the regulation of PASMC cycle proteins. (A) Representative western blots presenting the expression of Cyclin D1, Cyclin E, CDK1 and p27KIP in the four indicated experimental groups. (B) Quantified densitometry results of (A). Data are presented as the mean \pm standard deviation $(\mathrm{n}=10)$. ${ }^{*} \mathrm{P}<0.05$ vs. control; ${ }^{*} \mathrm{P}<0.05$ vs. shunt; and ${ }^{\$} \mathrm{P}<0.05$ vs. shunt + siRNA-TMEM16A. TMEM16A, transmembrane member 16A; PASMCs, pulmonary artery smooth muscle cells; siRNA, small interfering RNA; CDK1, cyclin-dependent kinase 1; p27KIP, cyclin-dependent kinase inhibitor P27.

pulmonary structural remodeling. In particular, TMEM16A may contribute to PAH induced by high blood flow by regulating the cell cycle progression of PASMCs.

Although TMEM16A has been extensively studied in cardiovascular diseases (15,19-21,23-25), conflicting results have been reported. Whilst certain studies have determined that the TMEM16A protein activated CaCCs in PASMC proliferation and spontaneous hypertension $(15,19,20)$, others have indicated that TMEM16A served an inhibitory role in the regulation of PASMC cell proliferation $(21,25)$. During the process of cerebral vascular remodeling of hypertension (21), the reduced expression of TMEM16A promoted $\mathrm{G}_{1}-\mathrm{S}$ phase cell cycle progression in cerebral arterial smooth muscle cells, indicating that TMEM16A is a negative regulator of cell proliferation in vascular smooth muscle cells. Expression of TMEM16A in the pulmonary arterial smooth muscle of rats has been demonstrated to be upregulated in monocrotaline-induced pulmonary hypertension (26) and chronic hypoxia-induced pulmonary hypertension (16). 
TMEM16A serves a vital role in the regulation of cell proliferation. The effect of TMEM16A on cell proliferation has varied in previous studies, and the reason for these contradictory results have not reached a unified conclusion. In an angiotensin II treatment-induced hypertension model, TMEM16A expression in basilar smooth muscle cells (BASMCs) was reduced (21), which was accompanied by an increased BASMC proliferation. Increased TMEM16A expression also promoted portal vein smooth muscle cell (PVSMC) proliferation in vitro (22), which is consistent with the results of the present study. TMEM16A was reported to be downregulated in PVSMCs from a rat model of portal hypertension, which resulted in increased PVSMC proliferation, indicating that angiotensin II may be a candidate regulatory factor for PASMC proliferation mediated by TMEM16A (22). In congenital hypertensive rats (20), hypoxia-induced and monocrotaline-induced pulmonary hypertension models $(14,15)$, the overexpression of TMEM16A promoted the proliferation of PASMCs. The present study determined that TMEM16A expression was upregulated in PASMCs from PAH induced by high blood flow.

Previous experiments have demonstrated that TMEM16A is closely associated with the expression of some cyclins $(27,28)$. In the present study it was determined that the expression of cyclin E and cyclin D1 increased in PASMCs from high blood flow-induced PAH. Cyclin D1 promotes cell cycle progression from the $G_{1}$ phase to the $S$ phase, whereas cyclin $E$ is essential for the normal development of the cell cycle (27). The complex of cyclin E and CDK2 (Cyclin E-CDK2) serves a crucial role in the process of entering the $S$ phase from the $G_{1}$ phase, and it is a key kinase complex regulator of the cell cycle. The overexpression of cyclin D1 may shorten the time required to enter $\mathrm{S}$ phase and accelerate the $\mathrm{G}_{1} / \mathrm{S}$ transition process, leading to cell proliferation (28). The siRNA-TMEM16A lentivirus was used to infect PASMCs isolated from rats that underwent PAH injury, where the decrease in cyclin D1 expression lead to cell cycle arrest at the $\mathrm{G}_{0}-\mathrm{G}_{1}$ phase, which inhibited cell proliferation and pulmonary vascular remodeling (29). The present study demonstrated increased expressions of cyclin D1 and cyclin E in PASMCs from PAH models, which was consistent with the results of a previous study (21). The p27 KIP family protein is a regulator of the cyclinE-CDK2 complex that inhibits cell proliferation by inhibiting cyclin E expression (30). As a negative regulatory factor of the cyclin E-CDK2 complex, the expression of p27 KIP was not affected by PAH, which is consistent with a previous study by Wang et al (21). In present study, although the expression of cyclin $\mathrm{E}$ in the PASMCs from shunt group increased compared with control group, the expression of CDK2 complexed to cyclin E in the shunt group did not change significantly compared with control PASMCs. The potential reason for this observation may be that the upregulation of TMEM16A during high pulmonary blood flow-induced PASMCs injury affected the expression of cyclin E and cyclin D1 via another paracrine mechanism without affecting the expression of p27 KIP and CDK2. It is also possible that the expression of CDK2 and p27 KIP remained relatively stable inside the cells but existed in an inactive form or were in complex with other proteins.

The present study has certain limitations. The recorded right heart pressure was mild and was limited to an early stage of high blood flow-induced PAH. In addition, studies addressing the TMEM16A-mediated regulation of protein expression that is associated with cell cycle progression, particularly the effect of TMEM16A knockout in vivo, are urgently required. Finally, specific signaling pathways, including ERK/cyclin D1 (31) and/or cyclin D1-MARK (32-34), serve as potential targets for future studies.

In conclusion, TMEM16A may be involved in high pulmonary blood flow-induced PAH by regulating cell cycle progression in PASMCs. TMEM16A may therefore serves as a novel therapeutic target for high pulmonary flow-induced PAH.

\section{Acknowledgements}

Not applicable.

\section{Funding}

The study was supported in part by the Natural ScienceFoundation of China (grant no. NSFC 81160040); Guangxi Natural Science Foundation (grant no. 2013GXNSFAA019177); Natural Science Foundation of Zhejiang Province (grant no. LY16H020010); Medicine Health Science and Technology Plan of Zhejiang Province (grant no. 2017194804) and Wenzhou Science and Technology Bureau, P.R. China (grant no. Y20160021).

\section{Availability of data and materials}

The datasets used and/or analyzed during the current study are available from the corresponding author on reasonable request.

\section{Authors' contributions}

LS and KW performed experiments and wrote the manuscript. DL, SQ, JH, YZ and YP contributed to data interpretation and discussion. YP conceived and directed the project, and edited the manuscript. YP is the guarantor of this project and, as such, has full access to all the data in the study and take responsibility for the integrity of the data and the accuracy of the data analysis. All authors read and approved the final manuscript.

\section{Ethics approval and consent to participate}

The present study was approved by The Ethics Committee of Guangxi Medical University (Nanning, China).

\section{Patient consent for publication}

Not applicable.

\section{Competing interests}

The authors declare that they have no competing interests.

\section{References}

1. Liu Y, Chen S, Zühlke L, Black GC, Choy MK, Li N and Keavney BD: Global birth prevalence of congenital heart defects 1970-2017: Updated systematic review and meta-analysis of 260 studies. Int J Epidemiol 48: 455-463, 2019. 
2. Li L, Jick S, Breitenstein S, Hernandez G, Michel A and Vizcaya D: Pulmonary arterial hypertension in the USA: An epidemiological study in a large insured pediatric population. Pulm Circ 7: 126-136, 2017.

3. Rubin LJ: Primary pulmonary hypertension. N Engl J Med 336: 111-117, 1997.

4. Huang F, Wong X and Jan LY: International Union of Basic and Clinical Pharmacology. LXXXV: Calcium-activated chloride channels. Pharmacol Rev 64: 1-15, 2012.

5. Hübner CA, Schroeder BC and Ehmke H: Regulation of vascular tone and arterial blood pressure: Role of chloride transport in vascular smooth muscle. Pflugers Arch 467: 605-614, 2015.

6. Yang Z, Zhang Z, Xu Y, Li Y and Ye T: Relationship of intracellular free $\mathrm{Ca}^{2+}$ concentration and calcium-activated chloride channels of pulmonary artery smooth muscle cells in rats under hypoxic conditions. J Huazhong Univ Sci Technolog Med Sci 26 172-174, 191, 2006.

7. Zaiman AL, Damico R, Thoms-Chesley A, Files DC, Kesari P, Johnston L, Swaim M, Mozammel S, Myers AC, Halushka M, et al: A critical role for the protein apoptosis repressor with caspase recruitment domain in hypoxia-induced pulmonary hypertension. Circulation 124: 2533-2542, 2011.

8. Caputo A, Caci E, Ferrera L, Pedemonte N, Barsanti C, Sondo E, Pfeffer U, Ravazzolo R, Zegarra-Moran O and Galietta LJ: TMEM16A, a membrane protein associated with calcium-dependent chloride channel activity. Science 322: 590-594, 2008.

9. Schroeder BC, Cheng T, Jan YN and Jan LY: Expression cloning of TMEM16A as a calcium-activated chloride channel subunit. Cell 134: 1019-1029, 2008.

10. Yang YD, Cho H, Koo JY, Tak MH, Cho Y, Shim WS, Park SP, Lee J, Lee B, Kim BM, et al: TMEM16A confers receptor-activated calcium-dependent chloride conductance. Nature 455 $1210-1215,2008$

11. Tian XQ, Ma KT, Wang XW, Wang Y, Guo ZK and Si JQ: Effects of the calcium-activated chloride channel inhibitors T16Ainh-A01 and CaCCinh-A01 on cardiac fibroblast function. Cell Physiol Biochem 49: 706-716, 2018.

12. Hyuga S, Danielsson J, Vink J, Fu XW, Wapner R and Gallos G Functional comparison of anoctamin 1 antagonists on human uterine smooth muscle contractility and excitability. J Smooth Muscle Res 54: 28-42, 2018.

13. Martinez-Pinna J, Soriano S, Tuduri E, Nadal A and de Castro F: A calcium-dependent chloride current increases repetitive firing in mouse sympathetic neurons. Front Physiol 9: 508, 2018.

14. Revermann M, Schloss M, Mieth A, Babelova A, Schröder K, Neofitidou S, Buerkl J, Kirschning T, Schermuly RT, Hofstetter C and Brandes RP: Levosimendan attenuates pulmonary vascular remodeling. Intensive Care Med 37: 1368-1377, 2011.

15. Sun H, Xia Y, Paudel O, Yang XR and Sham JS: Chronic hypoxia-induced upregulation of $\mathrm{Ca} 2+$-activated $\mathrm{Cl}$ - channel in pulmonary arterial myocytes: A mechanism contributing to enhanced vasoreactivity. J Physiol 590: 3507-3521, 2012.

16. Wang K, Ma J, Pang Y, Lao J, Pan X, Tang Q, Zhang F, Su D, Qin S and Shrestha AP: Niflumic acid attenuated pulmonary artery tone and vascular structural remodeling of pulmonary arterial hypertension induced by high pulmonary blood flow in vivo. J Cardiovasc Pharmacol 66: 383-391, 2015.

17. Lao J, Pang Y, Wang K, Liu D, Su D, Zhang F, Pan X and Li S: Inhibition of survivin promotes pulmonary arterial smooth muscle cell apoptosis in high blood flow-induced pulmonary arterial hypertension in rats. Int J Clin Exp Pathol 9: 6821-6834, 2016

18. Wang K, Chen C, Ma J, Lao J and Pang Y: Contribution of calcium-activated chloride channel to elevated pulmonary artery pressure in pulmonary arterial hypertension induced by high pulmonary blood flow. Int J Clin Exp Pathol 8: 146-154, 2015.

19. Manoury B, Tamuleviciute $A$ and Tammaro $P$. TMEM16A/anoctamin 1 protein mediates calcium-activated chloride currents in pulmonary arterial smooth muscle cells. J Physiol 588: 2305-2314, 2010.
20. Wang $\mathrm{B}$, Li $\mathrm{C}$, Huai $\mathrm{R}$ and $\mathrm{Qu} \mathrm{Z}$ : Overexpression of ANO1/TMEM16A, an arterial $\mathrm{Ca}^{2+}$-activated Cl- channel, contributes to spontaneous hypertension. J Mol Cell Cardiol 82: 22-32, 2015

21. Wang M, Yang H, Zheng LY, Zhang Z, Tang YB, Wang GL, Du YH, Lv XF, Liu J, Zhou JG and Guan YY: Downregulation of TMEM16A calcium-activated chloride channel contributes to cerebrovascular remodeling during hypertension by promoting basilar smooth muscle cell proliferation. Circulation 125: 697-707, 2012

22. Zeng X, Huang $P$, Chen M, Liu S, Wu N, Wang $F$ and Zhang J: TMEM16A regulates portal vein smooth muscle cell proliferation in portal hypertension. Exp Ther Med 15: 1062-1068, 2018

23. Dai WJ, Qiu J, Sun J, Ma CL, Huang N, Jiang Y, Zeng J, Ren BC, Li WC and Li YH: Downregulation of microRNA-9 reduces inflammatory response and fibroblast proliferation in mice with idiopathic pulmonary fibrosis through the ANO1-mediated TGF- $\beta$-Smad3 pathway. J Cell Physiol 234: 2552-2565, 2019.

24. Wu H, Wang H, Guan S, Zhang J, Chen Q, Wang X, Ma K, Zhao P, Zhao H, Yao W, et al: Cell-specific regulation of proliferation by Ano1/TMEM16A in breast cancer with different ER, PR, and HER2 status. Oncotarget 8: 84996-85013, 2017.

25. Zhang XH, Zheng B, Yang Z, He M, Yue LY, Zhang RN, Zhang M, Zhang W,Zhang X and Wen JK: TMEM16A and myocardin form a positive feedback loop that is disrupted by KLF5 during Ang II-induced vascular remodeling. Hypertension 66: 412-421, 2015.

26. Forrest AS, Joyce TC, Huebner ML, Ayon RJ, Wiwchar M, Joyce J, Freitas N, Davis AJ, Ye L, Duan DD, et al: Increased TMEM16A-encoded calcium-activated chloride channel activity is associated with pulmonary hypertension. Am J Physiol Cell Physiol 303: C1229-C1243, 2012.

27. Deng L, Yang J, Chen H, Ma B, Pan K, Su C, Xu F and Zhang J: Knockdown of TMEM16A suppressed MAPK and inhibited cell proliferation and migration in hepatocellular carcinoma. Onco Targets Ther 9: 325-333, 2016.

28. Liu J, Liu Y, Ren Y, Kang L and Zhang L: Transmembrane protein with unknown function $16 \mathrm{~A}$ overexpression promotes glioma formation through the nuclear factor- $\mathrm{\kappa B}$ signaling pathway. Mol Med Rep 9: 1068-1074, 2014.

29. Zeng DX, Xu GP, Lei W, Wang R, Wang CG and Huang JA: Suppression of cyclin D1 by plasmid-based short hairpin RNA ameliorated experimental pulmonary vascular remodeling. Microvasc Res 90: 144-149, 2013.

30. Liu P and Chen L: Inhibition of sonic hedgehog signaling blocks cell migration and growth but induces apoptosis via suppression of FOXQ1 in natural killer/T-cell lymphoma. Leuk Res 64: 1-9, 2018.

31. Li T, Song T, Ni L, Yang G, Song X, Wu L, Liu B and Liu C: The p-ERK-p-c-Jun-cyclinD1 pathway is involved in proliferation of smooth muscle cells after exposure to cigarette smoke extract. Biochem Biophys Res Commun 453: 316-320, 2014.

32. Qin L, Yang YB, Yang YX, Gong YZ, Li XL, Li GY, Luo HD, Xie XJ, Zheng XL and Liao DF: Inhibition of smooth muscle cell proliferation by ezetimibe via the cyclin D1-MAPK pathway. J Pharmacol Sci 125: 283-291, 2014.

33. Duvvuri U, Shiwarski DJ, Xiao D, Bertrand C, Huang X, Edinger RS, Rock JR, Harfe BD, Henson BJ, Kunzelmann K, et al: TMEM16A induces MAPK and contributes directly to tumorigenesis and cancer progression. Cancer Res 72: 3270-3281, 2012.

34. Song Y, Gao J, Guan L, Chen X, Gao J and Wang K: Inhibition of ANO1/TMEM16A induces apoptosis in human prostate carcinoma cells by activating TNF- $\alpha$ signaling. Cell Death Dis 9: 703, 2018.

This work is licensed under a Creative Commons Attribution-NonCommercial-NoDerivatives 4.0 International (CC BY-NC-ND 4.0) License. 